\title{
Limb edema in patients with advanced disease - a pilot study of compression therapy combined with diuretics
}

\begin{abstract}
Introduction. Management of edema due to advanced disease is seldom recognized in palliative care. The purpose of this pilot study was to assess the effectiveness and tolerance of conservative edema therapy. Material and methods. Prospective analysis of limb volumes, edema symptom burden and quality of life (QoL) in 119 patients diagnosed with edema was performed. Eighteen patients in their last days of life complaining of edema were treated by limb elevation, supportive (palliative) bandaging, manual lymphatic drainage, Kinesio Taping and/or controlled subcutaneous needle drainage. Forty-six with longer prognosis were treated by one-week multilayer short-stretch limb bandaging and then re-evaluated. Twenty-eight of them with venous congestion resistant to previously given diuretics received additionally furosemide infusion in hypertonic saline. Subgroups analysis of the patients with diuretic therapy and primary lymphatic congestion was performed.

Results. Within 46 patients re-evaluated the reduction of limb volume was accompanied by a decrease of edema symptom intensity and an improvement of QoL. Subgroup analysis revealed both volume and symptomatic improvement, equally effective in cases with suspected lymphatic congestion or when diuretics were added.

Conclusion. Edema of advanced disease irrespective of the lymphatic component can be effectively managed conservatively with short-stretch limb bandaging. In cases resistant to oral diuretic fluid overload, parenteral hypersaline furosemide addition to the compression may be the safe therapeutic option enhancing treatment tolerance.
\end{abstract}

Palliat Med Pract 2019; 13, 2: 51-56

Key words: advanced disease, diuretics, edema, furosemide, palliative care, quality of life

\section{Introduction}

Edema at the end of life population is a common harmful symptom, still seldom recognized. Despite typical presentation in the literature as lymphedema, it generally has a combined etiology (e.g. increased capillary hydrostatic, decreased plasma oncotic pressures, increased capillary permeability and lymphatic obstruction) [1]. The management engages the whole palliative multidisciplinary team [2]. Some case studies described various types of edema management. The most often recommended physiotherapeutic management strategies are characterized by poor tolerance [3]. The purpose of this paper, following recently published cross sectional study [4], was to assess the effectiveness of the intensive conservative management including short stretch compression bandaging broadened by additional diuretic treatment in cases

\footnotetext{
Address for correspondence:

Tomasz Grądalski

TPCH Hospicjum im. św. Łazarza w Krakowie/St Lazarus Hospice

Fatimska St. 17, 31-831 Kraków

e-mail: tomgr@mp.pl
}

Palliative Medicine in Practice 2019; 13, 2, 51-56

Copyright (C) Via Medica, ISSN 2545-0425

DOI: 10.5603/PMPI.2019.0009 
of general fluid congestion, provided within the hospice setting. Subgroups analysis of suspected primary lymphatic congestion origin edemas and the patients with diuretic addition was performed.

\section{Material and methods}

A prospective analysis of all patients with visible, spontaneously irreversible, chronic edema admitted to the free-standing hospice was performed. On admission, the edema localization and limb volumes were calculated by circumferential tape measurements. Additionally typically observed with edema sensations and overall wellbeing impairment (measured by the 5-item Likert scale: none, little, moderate, severe, very severe) were scored. The quality of life (QoL) according to Edmonton Symptom Assessment System-Core (ESAS-C) [5] was also monitored. ESAS-C is a shortened ESAS version designed for patients in their last days of life, with an improved completion rate. It equals the sum of five core symptom intensity measured on a numeric rating scale (NRS): pain, dyspnea, nausea, audible chest secretions and agitation (scores of each symptom range from 0 - the best to 10 - the worst, thus the total ESAS-C scores range from 0 to 50 ).

In those cases where the edema was noticed by the patients and caused discomfort, the 2-3 layers of short-stretch bandage compression (8 and $10 \mathrm{~cm}$ width, Rosidal K, Lohmann \& Rauscher, Germany) were applied by two skilled physiotherapists. Additionally, in patients with signs of systemic and/or pulmonary venous congestion, and when edema was resistant to previously given diuretics, parenteral furosemide (mean dose $60 \mathrm{mg}$, range 40-100 mg) was given over an hour in hypersaline intravenous infusion $(30 \mathrm{~mL}$ $10 \% \mathrm{NaCl}$ in $250 \mathrm{~mL}$ normal saline) concurrently with the compression, once daily. Routine daily clinical bedside physical evaluation of fluid congestion was performed. Later, after 5-7 days of such therapy, limb volumes, symptom intensity, well-being and QoL was re-evaluated. The study protocol was approved by the Local Ethics Committee.

\section{Statistical analysis}

Means with standard deviations (SD) of normally distributed continuous variables in one sample (before and after intervention) were compared using paired t-test. Wilcoxon signed rank test was used to compare medians with interquartile ranges (IQR) of non-normally distributed continuous or ordinal data in one sample. The association between the presence of pathognomic for the presence of lymphedema Stemmer Sign (inability to ,tent' the skin of the lymphedematous interdigital skin fold) and positive history of lymphatic impairment was calculated using McNemar's test. Comparison between subgroups was done using the Mann-Whitney $\mathrm{U}$ test (when comparing two variables) or the Kruskal-Wallis test (in four subgroups). A P the value of 0.05 was considered statistically significant. Data analysis was performed through the $R$ software environment for statistical computing and graphics software v. 3.5.0 [6].

\section{Results}

Among 119 patients (58.8\% females) who were diagnosed on admission with edema the majority had advanced cancer in Gold Standard Framework (GSF) [7] stage C and with bilateral edema, stage 2 according to the International Society of Lymphology. In 33 patients (27.7\%) the edema was the main problem on admission. The mean duration of hospice care was 11 days; 106 patients (89.1\%) eventually died. The leading causes of edema seen were chronic immobilization due to asthenia (95; $79.8 \%)$ and edema precipitating pharmacotherapy provided $(70 ; 58.8 \%)$. Fifty-six $(91.8 \%)$ of 61 patients clinically suspected of lymphedema had positive Stemmer sign [8], which contrasted to $11(19.0 \%)$ cases within the group who were not suspected of lymphatic congestion (Odds Ratio 0.36; Confidence Interval $0.08-1.22 ; \mathrm{p}=0.12$ ). Only 5 persons $(4.2 \%)$ received edema physiotherapy before the admission. Forty-one cases of edema (34.5\%) were not treated at the hospice, as this symptom was unnoticeable for the patients on examination (not being the source of suffering). Eighteen patients in their last days of life complaining of edema were qualified to individually tapered supportive management: limb elevation, supportive (palliative) compression bandaging (CB), manual lymphatic drainage (MLD), Kinesio Taping or controlled subcutaneous needle drainage and were not systematically re-evaluated.

Forty-six patients (38.6\%; 84 limbs in total), were managed by daily compression bandaging applied uniformly and re-evaluated. Eighteen patients (33 limbs) received diuretics additionally to compression. The edema with suspected concomitant lymphatic congestion was more advanced on admission, but the effect achieved through the two types of management appeared to be congruent (Table 1). Within all re-evaluated an excellent adherence to the prescribed treatment was seen: every patient fulfilled the strict scheduled 5-7 days management. There were no signs of additional venous fluid accumulation due to limb compression. All re-evaluated continued compression bandaging as needed ( 8 hours every $1-3$ days) up to 
Table 1. Limb volumes (L) and volume change within the managed group and subgroups

\begin{tabular}{|c|c|c|c|c|c|c|c|c|c|c|c|c|c|}
\hline \multirow{3}{*}{$\begin{array}{l}\text { Group/ } \\
\text { /Sub- } \\
\text { groups } \\
\text { Total }\end{array}$} & \multicolumn{2}{|c|}{ Patients } & \multicolumn{2}{|c|}{ Limbs } & \multicolumn{4}{|c|}{$\begin{array}{c}\text { Mean (SD) limbs volume } \\
\text { (L) }\end{array}$} & \multicolumn{4}{|c|}{$\begin{array}{l}\text { Mean (SD)/median (IQR) } \\
\text { limbs volume change }\end{array}$} & \multirow{3}{*}{$\begin{array}{c}p \\
<0.001^{*}\end{array}$} \\
\hline & \multirow{2}{*}{$\begin{array}{l}n \\
46\end{array}$} & \multirow{2}{*}{$\begin{array}{c}\% \\
100.0\end{array}$} & \multirow{2}{*}{$\begin{array}{l}n \\
84\end{array}$} & \multirow{2}{*}{$\begin{array}{c}\% \\
100.0\end{array}$} & \multicolumn{2}{|c|}{$\begin{array}{c}\text { On } \\
\text { admission }\end{array}$} & \multicolumn{2}{|c|}{$\begin{array}{l}\text { After } 5-7 \\
\text { days }\end{array}$} & \multicolumn{2}{|r|}{$\mathbf{L}$} & \multicolumn{2}{|c|}{$\begin{array}{c}\% \text { of initial } \\
\text { volume }\end{array}$} & \\
\hline & & & & & 6.66 & (3.14) & 5.48 & $(2.57)$ & -1.18 & (1.4) & -16.6 & $(14.1)$ & \\
\hline LE & 32 & 69.6 & 57 & 67.9 & 7.35 & (3.21) & 6.01 & $(2.58)$ & -1.35 & (1.54) & -17.2 & (13.9) & $<0.001^{*}$ \\
\hline \multirow[t]{2}{*}{$\mathrm{nLE}$} & 14 & 30.4 & 27 & 32.1 & 5.19 & $(2.43)$ & 4.37 & $(2.20)$ & $-0,82$ & $(0,95)$ & -15.5 & $(14.7)$ & $<0.001$ * \\
\hline & & \multicolumn{7}{|c|}{$p=0.001 \$$} & & \multicolumn{4}{|c|}{$p=0.3 \$$} \\
\hline CB & 28 & 60.9 & 51 & 60.7 & 7.18 & (3.28) & 5.93 & $(2.60)$ & -1.25 & $(1.60)$ & -15.5 & (14.9) & $<0.001^{*}$ \\
\hline \multirow[t]{2}{*}{ CBD } & 18 & 39.1 & 33 & 39.3 & 5.85 & $(2.76)$ & 4.78 & (2.39) & -1.07 & $(1.03)$ & -18.4 & (12.7) & $<0.001$ * \\
\hline & & \multicolumn{7}{|c|}{$p=0.17 \$$} & & \multicolumn{4}{|c|}{$p=0.8 \$$} \\
\hline $\begin{array}{l}\mathrm{LE}+ \\
\mathrm{CBD}\end{array}$ & 11 & 23.9 & 19 & 22.6 & 7.33 & (2.54) & 6.14 & $(2.16)$ & -1.19 & (1.19) & 16.4 & $(12.8)$ & $<0.001 *$ \\
\hline $\begin{array}{l}\mathrm{nLE}+ \\
\mathrm{CBD}\end{array}$ & 7 & 15.2 & 14 & 16.7 & 3.84 & $(1.50)$ & 2.93 & $(1.11)$ & -0.90 & $(0.77)$ & 21.2 & $(12.5)$ & $<0.001 *$ \\
\hline $\mathrm{LE}+\mathrm{CB}$ & 21 & 45.7 & 38 & 45.2 & 7.37 & (3.53) & 5.94 & (2.79) & -1.43 & $(1.70)$ & 7.6 & $(14.6)$ & $<0.001^{*}$ \\
\hline \multirow[t]{2}{*}{$\mathrm{nLE}+\mathrm{CB}$} & 7 & 15.2 & 13 & 15.5 & 6.64 & $(2.44)$ & 5.91 & $(2.04)$ & -0.76 & $\begin{array}{l}(-1.22 \text { to } \\
-0.42)\end{array}$ & 10.5 & $\begin{array}{l}(-4.8 \text { to } \\
-20.0)\end{array}$ & $0.02^{* *}$ \\
\hline & & \multicolumn{7}{|c|}{$p=0.07 \#$} & & \multicolumn{4}{|c|}{$p=0.3 \#$} \\
\hline
\end{tabular}

LE - edema of lymphatic etiology; nLE - non-lymphatic etiology; CB - compression bandaging; CBD — compression bandaging and diuretics Significance within the group ( ${ }^{*}$ t-test; ${ }^{*}$ Wilcoxon Signed Rank test); significance between subgroups (\$Mann-Whitney U test; \#Kruskal-Wallis test)

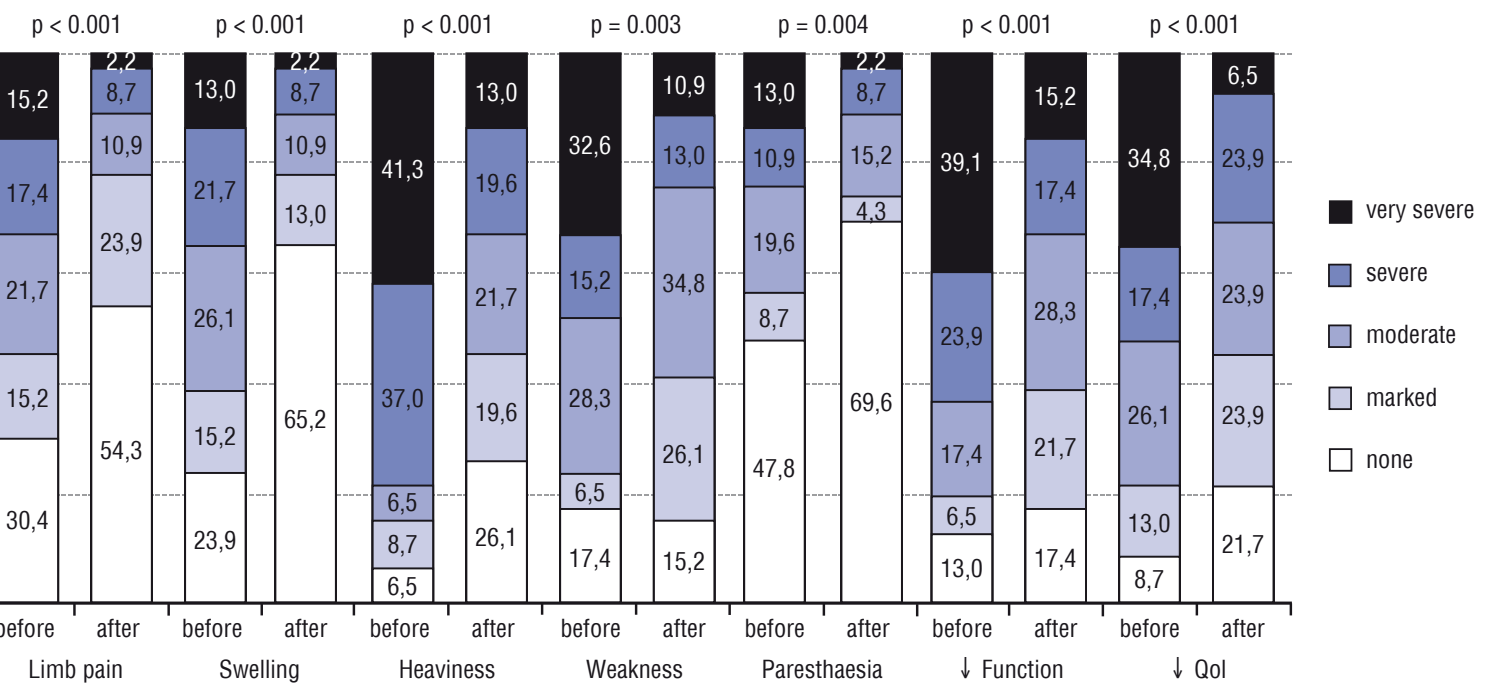

Figure 1. The percentage of the intensity of commonly seen edema symptoms and QoL impairment according to the Likert 5-item scale on admission (before) and after 5-7 days of the management within the group of 46 patients (Wilcoxon Signed Rank Test)

the dying phase or hospice discharge - diuretic subgroup with oral torsemide (range 2.5-10 mg a day).

The intensity of commonly seen edema symptoms and overall well-being impairment improved in a short time within the whole group of patients (Fig. 1).
The sum of edema sensations severity in all those treated decreased from 17 (IQR 11-20) to 7 (5-15); $p<0.001)$. The median total ESAS-C score decreased from 25 to $16(p=0.002)$. The subgroup analysis revealed symptomatic improvement in edema sensations 
and limb heaviness in every subgroup but analyzed symptom changes correlated neither with the edema etiology nor with the management intensity (Table 2 )

\section{Discussion}

The typical feature seen on hospice admission was bilateral lower limbs mixed etiology disease involvement, that confirmed previous observations [9]. Nearly two-thirds revealed late stages of edema which are more problematic to manage. The suspicion of lymphatic congestion being the primary cause of edema was made on the basis of a history of iatrogenic oncological treatment. Positive Stemmer sign made diagnosis more presumable but positive test in 11 cases without a history of lymphatic damage indicated secondary lymphatic insufficiency. Despite the presence of cancer in nearly all cases, non-lymphatic edema etiology (relative fluid overload, low plasma oncotic pressure, or/and elevated venous pressure) seemed to be of equal importance. In these situations, capillary filtration overwhelmed the lymph drainage for a significant period of time. The lymph transport which ensures the interstitial fluid drainage cannot be maintained indefinitely. At first, the increased lymphatic load prevails the lymphatic physiological outflow capacity, which leads to functional insufficiency. This longer lasting process promotes inflammation, tissue fibrosis and lymphatic congestion - condition similar that is in lymphedema. In practice, most edema seen are compound of origin, and when untreated they consisted of both lymphatic congestive failure and high microvascular fluid filtration [10].

The drugs typically used to treat edema prior to palliative care were diuretics, however, they were insufficient in this group, probably also due to lowered oncotic pressure in hypoalbuminemia [11]. This kind of therapy can be even counterproductive, as diuretics diminish water and electrolytes in the edematous tissue, leaving cells and proteins, thus promoting earlier connective/fat formation. Clinicians should take into account the plethora of side effects of these drugs profoundly diminish the patient's QoL. The primary aim of palliative care is to improve comfort measures, increasing/maintaining functional capacity and enhancing QoL, rather than to resolve the swelling completely. Therefore, any treatment that may be burdensome with minimal benefit should be avoided [12].

Various therapeutic modalities are proposed to palliative care patients with edema. The standard physical therapy for these patients is still being debated, as controlled studies are lacking. When life prognosis is short and edema quite stable, with low risk of further progression limb elevation, MLD [13] or Kinesio
Taping are preferred. In more advanced cases the trial of palliative $C B$ or controlled subcutaneous needle drainage can be initiated [14]. Unfortunately, in this study, the effectiveness of these techniques could not be assessed due to high attrition rate (14 patients died within a week). A substantial number of palliative care patients can tolerate only these methods that may have a beneficial role in symptom or QoL improvement despite an unproven value in edema reduction [15]. In patients with longer prognosis standard [16] or modified [3] programs of complex decongestive therapy (CDT) adapted to the patient's reduced treatment tolerance should be considered [17]. Short-stretch CB seems to be the most reducing limb volume component of CDT as MLD may not be necessary for limb volume reduction [18]. It is characterized by high working and low resting pressures, well tolerated and safer in moderately impaired arterial circulation or in chronic compensated heart failure [19]. However, careful consideration of the compression risk factors (further compromise arterial inflow or signs of additional venous fluid accumulation) should be monitored.

$\mathrm{CB}$ may not be well tolerated in palliative care population, thus in this trial, a combination of compression with pharmacotherapy was considered when fluid congestion was diagnosed. Combining parenteral diuretics with limb compression was previously shown to be safe in the case series of 19 patients [20]. Stable levels of blood pressure, laboratory kidney profiles (potassium, sodium, creatinine clearance) and serum albumin were also seen. In the current study, any signs of increasing fluid congestion (e.g. jugular veins distension, ascites progression or new edema, in particular genital, onset) were not seen. The symptom profile and QoL improvement in diuretic subgroup were similar to patients without diuretics administration. Furosemide before the hospice admission usually administered orally failed to adequately control salt and water retention despite dose escalation [21]. One of the strategies for overcoming this resistance is adding hypertonic saline solution [22] that probably restore the effective arterial volume and improve neurohormonal inhibition and renal hemodynamics [23]. However, possible short term risk factors of diuretics (e.g. acute renal insufficiency, electrolytes imbalance or thrombosis) should be also taken into account.

In this study the achieved positive effects in terms of limb volume reduction and decreasing edema symptom load which were visible regardless of the etiology of the disease or the diuretic addition. Reasonable patients' compression tolerance in this study, despite initially visible fluid congestion, suggests the beneficial clinical effect of added furosemide in the hypersaline infusion. Questions remain about the optimal use of diuretics 


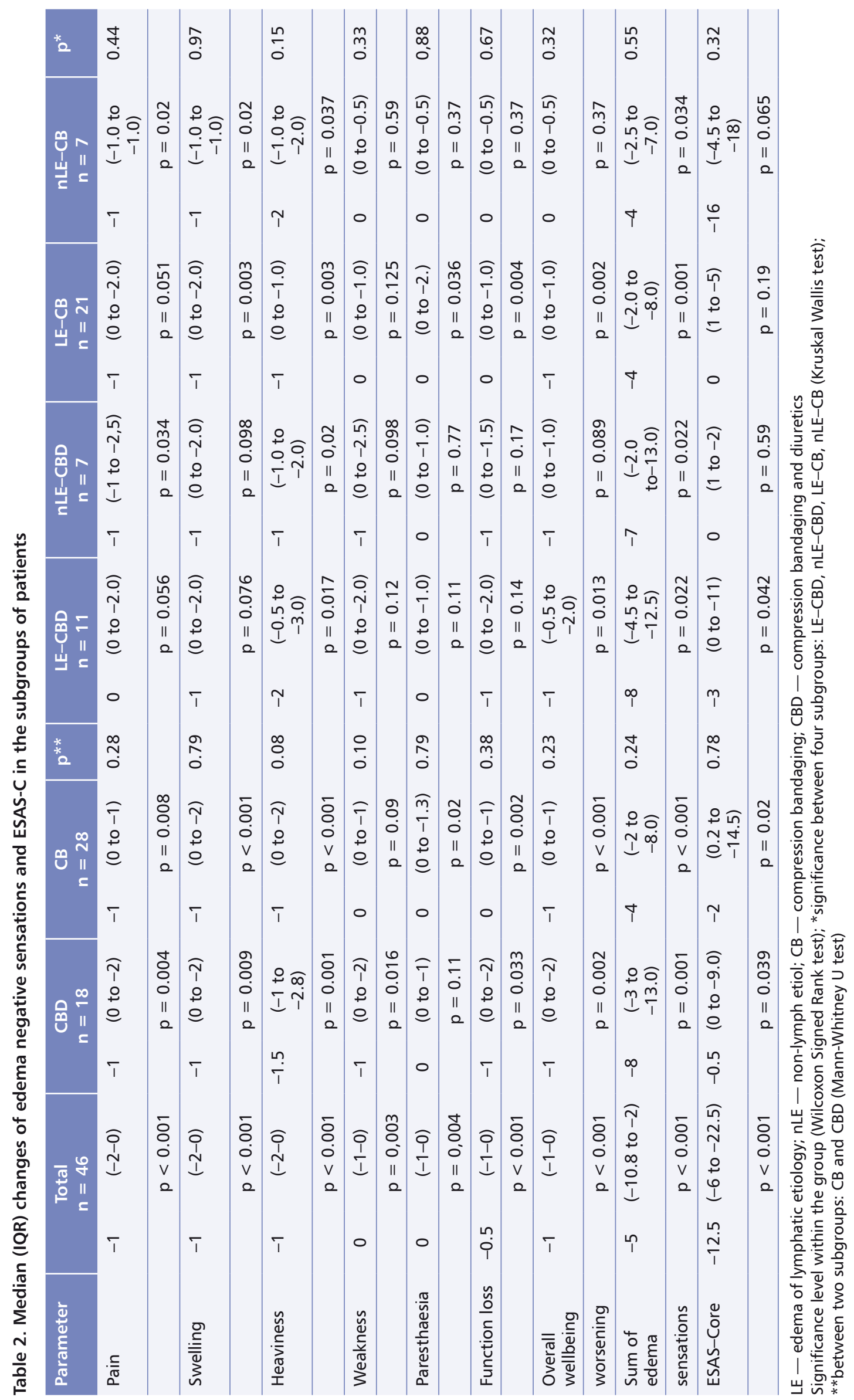


(avoiding excessive treatment) to obtain efficient decongestion. In the hospice setting simple physical examination monitoring of the neck veins and blood pressure are valuable [21]. In addition, the serial measures of hematocrit levels, indicating for the adequacy of fluid refilling rate from the extravascular to the vascular compartment could be helpful [22]. Compression limb bandaging, in turn, added to diuretics may promote the compensation of an excessively fast vessel fluid withdrawal. This small sample size and observational type of the study, limited to those who had been admitted to a single free-standing hospice, however, need to be validated in larger, randomized trials.

\section{Conclusions}

Edema in patients with advanced diseases is a polietiology group of diseases, including lymphatic congestion. It is a common source of suffering that can be effectively managed conservatively with short-stretch limb bandaging. In cases of edema in patients with resistance to oral diuretics fluid overload, parenteral hypersaline furosemide addition to the compression can facilitate this treatment tolerance and may be the safe therapeutic option.

\section{References}

1. Shah MG, Cho S, Atwood JE, et al. Peripheral edema. Am J Med. 2002; 113(7): 580-586, indexed in Pubmed: 12459405.

2. Olsson Möller U, Stigmar K, Beck I, et al. Bridging gaps in everyday life - a free-listing approach to explore the variety of activities performed by physiotherapists in specialized palliative care. BMC Palliat Care. 2018; 17(1): 20, doi: 10.1186/s12904-018-0272-x, indexed in Pubmed: 29378566.

3. Cobbe S, Nugent K, Real S. Pilot Study: The Effectiveness of Complex Decongestive Therapy for Lymphedema in Palliative Care Patients with Advanced Cancer. J Palliat Med. 2018; 21(4): 473-478, doi: 10.1089/jpm.2017.0235, indexed in Pubmed: 29206073.

4. Gradalski T. Edema of Advanced Cancer: Prevalence, Etiology, and Conservative Management-A Single Hospice Cross-Sectional Study. J Pain Symptom Manage. 2019; 57(2): 311-318, doi: 10.1016/j.jpainsymman.2018.11.005, indexed in Pubmed: 30453053.

5. Reid C, Gibbins J, Burcombe M, et al. The use of the edmonton symptom assessment scale to measure symptoms at the end of life. BMJ Supportive \& Palliative Care. 2012; 2(Suppl 1): A29.2-A29, doi: 10.1136/bmjspcare-2012-000196.85.

6. URL https://www R-project org. R: A language and environment for statistical computing. R Foundation for Statistical Computing, Vienna, Austria. (2017)

7. Gold Standards Framework. http://www. goldstandardsframework.org.uk/ (15.05.2018).

8. Stemmer R. Ein klinisches Zeichen zur Früh- und Differentialdiagnose des Lymphödems. Vasa. 1976; 5: 261-262.

9. Real S, Cobbe S, Slattery S. Palliative Care Edema: Patient Population, Causal Factors, and Types of Edema Referred to a Specialist Palliative Care Edema Service. J Palliat Med. 2016; 19(7): 771-777, doi: 10.1089/jpm.2015.0337, indexed in Pubmed: 27078226.

10. Moffatt CJ, Doherty DC, Franks PJ, et al. Community-Based Treatment for Chronic Edema: An Effective Ser- vice Model. Lymphat Res Biol. 2018; 16(1): 92-99, doi: 10.1089//rb.2017.0021, indexed in Pubmed: 29432067.

11. Towers A, Hodgson P, Shay C, et al. Care of palliative patients with cancer-related lymphoedema. J Lymphoedema. 2010; 5(1): 72-80.

12. Beck $M$, Wanchai $A$, Stewart $B R$, et al. Palliative care for cancer-related lymphedema: a systematic review. J Palliat Med. 2012; 15(7): 821-827, doi: 10.1089/jpm.2011.0494, indexed in Pubmed: 22662959.

13. Müller M, Klingberg K, Wertli MM, et al. Manual lymphatic drainage and quality of life in patients with lymphoedema and mixed oedema: a systematic review of randomised controlled trials. Qual Life Res. 2018; 27(6): 1403-1414, doi: 10.1007/s11136-018-1796-5, indexed in Pubmed: 29404923.

14. Jacobsen J, Blinderman CD. Subcutaneous lymphatic drainage (lymphcentesis) for palliation of severe refractory lymphedema in cancer patients. J Pain Symptom Manage. 2011; 41(6): 1094-1097, doi: 10.1016/j.jpainsymman.2010.09.015, indexed in Pubmed: 21402464.

15. Clemens KE, Jaspers B, Klaschik E, et al. Evaluation of the clinical effectiveness of physiotherapeutic management of lymphoedema in palliative care patients. Jpn J Clin Oncol. 2010; 40(11): 1068-1072, doi: 10.1093/jjco/hyq093, indexed in Pubmed: 20558463.

16. Gradalski T, Ochalek K, Rybak D. Lymphedema or rather end-of-life edema? J Palliat Med. 2018; 21(5): 585, doi: 10.1089/jpm.2017.0714.

17. Cheville AL, Andrews K, Kollasch J, et al. Adapting lymphedema treatment to the palliative setting. Am J Hosp Palliat Care. 2014; 31(1): 38-44, doi: 10.1177/1049909112475297, indexed in Pubmed: 23423772.

18. Gradalski T, Ochalek K, Kurpiewska J. Complex Decongestive Lymphatic Therapy With or Without Vodder II Manual Lymph Drainage in More Severe Chronic Postmastectomy Upper Limb Lymphedema: A Randomized Noninferiority Prospective Study. J Pain Symptom Manage. 2015; 50(6): 750-757, doi: 10.1016/j.jpainsymman.2015.06.017, indexed in Pubmed: 26303187.

19. Andriessen A, Apelqvist J, Mosti G, et al. Compression therapy for venous leg ulcers: risk factors for adverse events and complications, contraindications - a review of present guidelines. J Eur Acad Dermatol Venereol. 2017; 31(9): 1562-1568, doi: $10.1111 / \mathrm{jdv} .14390$, indexed in Pubmed: 28602045.

20. Gradalski T. Diuretics Combined With Compression in Resistant Limb Edema of Advanced Disease-A Case Series Report. J Pain Symptom Manage. 2018; 55(4): 1179-1183, doi: 10.1016/j. jpainsymman.2017.12.481, indexed in Pubmed: 29288880.

21. Won S, Youb S. Loop diuretics in Clinical Practice. Electrolyte Blood Press. 2015; 13: 17-21.

22. Tuttolomondo A, Di Raimondo D, Bellia C, et al. Immune-Inflammatory and Metabolic Effects of High Dose Furosemide plus Hypertonic Saline Solution (HSS) Treatment in Cirrhotic Subjects with Refractory Ascites. PLoS One. 2016; 11(12): e0165443, doi: 10.1371/journal.pone.0165443, indexed in Pubmed: 27941973

23. De Vecchis R, Esposito C, Ariano C, et al. Hypertonic saline plus i.v. furosemide improve renal safety profile and clinical outcomes in acute decompensated heart failure: A meta-analysis of the literature. Herz. 2015; 40(3): 423-435, doi: 10.1007/s00059-013-4041-6, indexed in Pubmed: 24682291.

24. Palazzuoli A, Ruocco G, Ronco C, et al. Loop diuretics in acute heart failure: beyond the decongestive relief for the kidney. Crit Care. 2015; 19: 296, doi: 10.1186/s13054-0151017-3, indexed in Pubmed: 26335137.

25. Marenzi G, Lauri G, Grazi M, et al. Circulatory response to fluid overload removal by extracorporeal ultrafiltration in refractory congestive heart failure. J Am Coll Cardiol. 2001; 38(4): 963-968, indexed in Pubmed: 11583865. 